\title{
Limitations and perspectives with the approach to rheumatic fever and rheumatic heart disease
}

\author{
Cleonice de C. Mota \\ Departamento de Pediatria, Faculdade de Medicina-Universidade Federal de Minas Gerais, Belo Horizonte, Brazil
}

\section{The impact of the epidemiological data}

Rheumatic fever, and the rheumatic heart disease which follows it, represent a health burden worldwide. If we take into consideration that developing countries, where the prevalence of the disease is high, contain the majority of the population in the world, we can see that the burden is far from inconsiderable. In these communities, the acute episodes of rheumatic fever are still a cause of death in childhood, and the valvar sequels of the disease are the most representative cause of acquired cardiac disease in children and young adults.

According to the World Health Organisation, an estimated 12 million individuals suffer from rheumatic fever and rheumatic heart disease, of whom at least one quarter have heart failure demanding repeated hospitalisation and surgical intervention within 5 to 10 years. ${ }^{1}$

As the disease is a condition carried throughout life, it has repercussions for patients of all ages. The children and adolescents, who contribute to hospital admissions due to the higher frequency of acute episodes in this young population, are the same groups which, after the age of 40, will invert the age distribution when the analysis involves intervention and death.

Even considering the increasingly detailed knowledge gathered from clinical experience and scientific investigation, this preventable disease remains a moving target, clinically and socially devastating. The financial burden related to the care needed by the patients represents an additional challenge. In areas where resources are limited, the economic impact must

Correspondence to: Cleonice de C. Mota, Chefe do Departamento de Pediatria, Faculdade de Medicina-Universidade Federal de Minas Gerais, Av. Prof. Alfredo Balena, $110-4^{\circ}$ andar, 30130-100 - Belo Horizonte - MG, Brazil. Tel: +55 31 324894 37; Fax: +55 313248 9770; E-mail: cleomota@medicina.ufmg.br be taken into account, not only with regard to the costs of clinical and surgical treatment, but also the loss of productivity as the result of a disability acquired at an early age. On a global scale, it has been estimated that, each year, at least one-third of a million patients die, and that 6.6 million disability-adjusted life years are lost to rheumatic heart disease. ${ }^{1}$

\section{Limitations to the approach}

It is a fact that continuing progress in the knowledge of rheumatic fever has been generated by basic and applied research, but the precise pathogenetic mechanisms have not been completely understood. Even considering the results focusing on appropriate primary and secondary prophylaxis, then as stated by Markowitz "there are obvious limitations in our ability to completely eradicate rheumatic fever with antibiotics". The outbreaks reported in the United States of America throughout the last two decades, along with those encountered more recently in Europe, have drawn attention to the potential resurgence of the disease in regions where it had virtually disappeared. ${ }^{3,4}$

Histological hallmarks of rheumatic fever, such as Aschoff nodules, can be identified at autopsy, and also through invasive investigation by, for example, right ventricular endomyocardial biopsy. On the other hand, in the setting of clinical and laboratory diagnoses, the lack of pathognomonic symptoms, and signs or specific tests, is well known. In addition, due to modifications in the human susceptibility, rheumatogenic potential of the bacteria, and changes in the environment, non-classical and mild forms of presentation have also contributed to making the diagnosis difficult. Mild carditis could remain clinically unrecognised, and the patient unprotected. Consequently, the risk of having the valvar lesions 
worsened by recurrences is higher. Unfortunately, even in the patients diagnosed by echocardiography as having subclinical valvitis or chronic subclinical valvar disease, the approach regarding the duration of secondary prophylaxis is undefined, and this issue remains controversial. ${ }^{5}$ At the moment, it seems reasonable to recognize the same potential risks for the patients with subclinical valvar lesions as much as for those with clinical evidence of carditis, and then to provide similar regimes of prevention for both groups of patients. ${ }^{6}$

Although the palliative procedures can improve the clinical condition, it is a fact that there is not a curative treatment in the approach of the rheumatic fever when the heart is involved. The evidence pointing to the inflammatory characteristics of the disease has given support for the use of anti-inflammatory agents. The treatment is symptomatically beneficial, but it does not prevent or reduce the severity of the valvar sequels in the long-term, and the comparison of the efficacy of one drug over others remains unclear. ${ }^{7}$

Since the initial surgical procedures on the cardiac valves undertaken in the late 1940s, the continuing advances in surgical techniques, prosthetic devices, and interventional catheterisation, besides those in peri- and post-operative care, have revolutionized the management, and improved the survival and quality of life of many patients with valvar disease. Considering the severity of the clinical condition, mainly due to acute regurgitation, surgery could be mandatory as a life-saving procedure. As the last step in the approach, the prevention of a fatal evolution and the recovery of patients by surgery have been considered by some as a tertiary prophylaxis. Even taking into account the benefits of surgical intervention, major clinical problems have to be dealt with when contemplating surgery in children and adolescents. The definition of the optimal timing for surgery remains uncertain for these age groups. Particulars, such as the pattern of growing-up, and the changing metabolism, besides the expectancy of longevity, may constitute factors favouring surgical intervention.

Reconstructive surgery is highly desirable in the young, but depending on severity of the lesions, the valvar repair could be technically challenging, and small patients submitted to valvar replacement may outgrow the prosthesis, thus necessitating reoperation. The limited durability of bioprotheses, due to early calcific degeneration, remains an ongoing problem, as do the complications involved with anticoagulation in children, mainly in the communities where living conditions and medical facilities are substandard.

As regards recurrences, and their adverse effects on clinical and surgical outcomes, developing communities have to face an additional challenge as a result of the socioeconomic and environmental context. Unfavourable conditions, such as overcrowding, with closer interpersonal contact, increase the risk of subsequent attacks. In a long-term follow-up from 2 to 15 years of 258 Brazilian children and adolescents since their first attack, the increased risk of progressing to severe chronic valvar disease was associated with recurrences, severity of the carditis, and the maternal level of education. ${ }^{8}$ According to our recent investigation of 63 patients under 20 years of age, submitted to repair of the mitral valve, we obtained an actuarial survival rate of $91.8 \%$ over 14 years. Recurrences of rheumatic fever among those patients were identified as an independent predictor of surgical failure (Dobrianskyj and Mota, unpublished data).

\section{The role and clinical application of echocardiography}

Since the Jones criterions were proposed, no method of investigation has made a greater contribution, to our knowledge, than Doppler echocardiography as regards the spectrum of cardiac damage due to rheumatic fever and rheumatic heart disease. This noninvasive technique has added important information about the functional and anatomic subtract of valvar and subvalvar apparatus, resulting in a better understanding of mechanisms of valvar dysfunction. This more accurate assessment has provided new insights to the role played by both myocarditis and valvitis in the clinical presentation of carditis, giving support for the introduction of a new physiopathologic concept. Despite the evidence of histopathological abnormalities of the myocardial involvement, the observations are consistent with a closer relation of the heart failure and ventricular dilation with the mechanical factor rather than the myocardial one, pointing towards the dominant role of the valvar lesion in the acute phase. ${ }^{9-11}$

The continuing evolution of technology after the advent of M-mode interrogation has allowed incorporation of new modalities, enhancing their sensitivity and specificity. As a consequence, the technique has provided a more accurate assessment of all degrees of severity, including an earlier diagnosis of the cardiac involvement. In this matter, the more representative conditions are the subclinical valvar lesions, both in the acute and chronic phases. On the other hand, echocardiography could contribute in the differential diagnosis by the exclusion of conditions such as congenital cardiac malformations, which in a clinical setting have been first diagnosed as rheumatic fever. Although Doppler echocardiography does not confirm the aetiology, and its findings must be interpreted in the clinical context, it 
is a powerful tool to support and guide decisions regarding management. The possibility of monitoring the progress of valvar damage is important in delineating the best timing for the interventional approach and the choice of procedure, either balloon valvoplasty or surgery. Additionally, the echocardiographic data has been used as a prognostic predictor of surgical results, while intraoperative transoesophageal echocardiography has facilitated advances in reconstructive valvar surgery. The more recent introduction of three-dimensional echocardiography has provided insights in the geometry and spatial relations of the valvar apparatus. ${ }^{12-14}$

\section{Perspectives}

Currently, our best perspectives in the approach to rheumatic fever include the search for a reliable genetic marker of susceptibility, which will allow the identification of individuals at risk of developing the disease, and the availability of an effective streptococcal vaccine to confer immunity, and consequently protect those individuals.

It is into this scene of limitations, and the need to improve our perspectives through research, that three papers on rheumatic fever published in this issue of Cardiology in the Young are most welcome. From a historical perspective of the evolution of knowledge, the detailed and well described review of Veasy and Tani $^{15}$ analyses the supporting evidence of the new concept for acute rheumatic mitral regurgitation, highlighting the discussion of the physiopathologic mechanisms and their interaction with the valvar structure and clinical presentation of the disease. Likewise, Aziz ${ }^{16}$ contributes to the clinical decision with the introduction of a new echocardiographic index addressed to support the determination of the optimal timing for the surgical intervention in children and adolescents. Vijayalakshmi et al., ${ }^{17}$ by investigating a large number of patients, bring the role played by echocardiography to the top of the discussion in both conditions, namely the under diagnosis and over diagnosis of rheumatic carditis, and the need to improve the diagnostic precision.

Considering that strict echocardiographic criterions are required, the more recent data concerning the application of echocardiography have been pointing towards the conclusion that the risks for patients with unrecognised valvitis are greater than the risks of over-diagnosis. Even taking into account the economic impact, this technological advance should be incorporated into clinical practice.

Until we answer both the old and the new questions, rheumatic fever will continue to puzzle clinicians and challenge the scientific community. Based on the accumulated experience, continuing attention must be given to implement the adherence of patients to regimes of prevention, while strategies must be tailored to the different epidemiological contexts. Looking forward for further progress, we must turn to scientific investigation as the major source of advances.

\section{References}

1. World Health Organization. Rheumatic Fever and Rheumatic Heart Disease. Report of a WHO Expert Consultation. WHO technical report series, 923, Geneva, 2004.

2. Markowitz M. Rheumatic fever - a half-century perspective. Pediatrics 1998; 102: 272-274.

3. Veasy LG, Tani LY, Daly JA, et al. Temporal association of the appearance of mucoid strains of Streptococcus pyogenes with the continuing high incidence of rheumatic fever in Utah. Pediatrics 2004; 113: 168-172.

4. De Santis M, Fesslova V, Salice S, et al. A possible comeback of rheumatic fever in Northern Italy. Ital J Pediatr 2003; 29: 217-221.

5. Ferrieri P, Baddour L, Bolger A, et al. Proceedings of Jones Criteria Workshop (AHA Scientific Statement). Committee on Rheumatic Fever, Endocarditis, and Kawasaki Disease of the American Heart Association. Circulation 2002; 106: 2521-2523.

6. Mota CC. Doppler echocardiography assessment of subclinical valvitis in the diagnosis of acute rheumatic fever. Cardiol Young 2001; 11: 251-254.

7. Albert DA, Harel L, Karrison T. The treatment of rheumatic carditis: review and metanalysis. Medicine 1995; 74: 1-12.

8. Meira ZMA, Goulart EMA, Colossimo EA, Mota CC. Long term follow up of rheumatic fever and predictors of severe rheumatic valvar disease in Brazilian children and adolescents. Heart 2005; 91: 1019-1022.

9. Essop MR, Wisenbaugh T, Sareli P. Evidence against a myocardial factor as a cause of left ventricular dilatation in active rheumatic carditis. J Am Coll Cardiol 1993; 22: 826-829.

10. Vasan RS, Shrivastava S, Vijayakumar M, Narang R, Lister BC, Narula J. Echocardiographic evaluation of patients with acute rheumatic fever and rheumatic carditis. Circulation 1996; 94: 73-82.

11. Kamblock J, Payot L, Lung B, et al. Does rheumatic myocarditis really exist? Systematic study with echocardiography and cardiac troponin I blood levels. Eur Heart J 2003; 24: 855-862.

12. Enriques-Sarano M, Tajik AJ, Schaff HV, Orszulak TA, Bailey KR, Frye RL. Echocardiographic prediction of survival after surgical correction of organic mitral regurgitation. Circulation 1994; 90: 830-837.

13. Bach DS, Deeb GM, Bolling SF. Accuracy of intraoperative transesophageal echocardiography for estimating the severity of functional mitral regurgitation. Am J Cardiol 1995; 76: 508-512.

14. Otsuji Y, Handsschumacher MD, Schwammenthal E, et al. Insights from three-dimensional echocardiography into mechanism of functional mitral regurgitation. Circulation 1997; 96: 1999-2008.

15. Veasy LG, Tani LY. A new look at acute rheumatic mitral regurgitation. Cardiol Young 2005; 15: 568-577.

16. Aziz K. An echocardiographic index for decompensation of the chronically volume-overloaded left ventricle in children. Cardiol Young 2005; 15: 589-596.

17. Vijayalakshmi IB, Mithravinda J, Prabhu Deva AN. The role of echocardiography in diagnosing carditis in the setting of acute rheumatic fever. Cardiol Young 2005; 15: 583-588. 